\title{
Effect Analysis of Hospital Nursing Management Based on Informatization
}

\author{
Lijuan Zhang*, Ling Sun, Huifang Cao, Shaofeng Zhu \\ Qianwei Hospital of Jilin Province, Changchun 130017, Jilin Province, China \\ *Corresponding author: Lijuan Zhang, rongguang-163@163.com
}

\begin{abstract}
Objective: To explore the effect of hospital nursing management based on informatization. Methods: 167 inpatients from different departments in our hospital from June 2020 to November 2019 were randomly selected and divided into control group and observation group. 83 cases in the control group were treated with traditional nursing management mode; 87 cases in the observation group used modern nursing information management system for information-based nursing management, and then compared the two groups in terms of nursing management efficiency, patient basic nursing, ward management, specialized nursing and nursing satisfaction, so as to verify the clinical application effect of information-based nursing management. Results: After the application of information-based nursing management mode, the construction and application of information management system made the nursing management process more standardized and accurate. Therefore, the clinical nursing efficiency, basic nursing, ward management, specialized nursing and nursing satisfaction of the observation group were better than those of the control group. Conclusion: In the practice of hospital inpatient nursing management, the scientific use of information technology and information management system can significantly improve the efficiency of patient nursing management and nursing satisfaction, and play a key role in improving the prognosis of patients. Therefore, it is worth popularizing and applying in the whole hospital and even the national grass-roots hospitals.
\end{abstract}

Keywords: Nursing management; Promotion of information technology; Nursing efficiency

Publication date: September 2021; Online publication: September 30, 2021

\section{Introduction}

With the increasing investment of the National Health Management Commission in grass-roots hospitals and the further improvement of the national scientific and technological level, hospital informatization has achieved all-round development. Information management systems based on emerging technologies such as Internet of things, big data and cloud computing are widely used in hospital nursing management, On the basis of boosting the optimization of hospital management process, it has effectively promoted the improvement of China's medical service level. In order to explore the innovative model and application effect of information-based nursing management in grass-roots hospitals, Chen Guanglan ${ }^{[1]}$ and others randomly selected 230 patients from different departments of a hospital for hospitalization, divided them into research group and control group, and then applied traditional nursing management and informationbased nursing management to the two groups of research objects respectively, By comparing the incidence of adverse events and the mastery of nursing knowledge under different management methods, it was found that the study was significantly better than the control group, which fully verified the great clinical application value of information-based nursing management mode. Through clinical trial research, 167 subjects were treated with different nursing management modes, and then the clinical effects of the two management modes were compared. The specific research process and results are reported as follows: 


\section{Data and methods}

\subsection{Clinical data}

167 inpatients under nursing management in our hospital from June 2020 to November 2019 were randomly selected and divided into two groups by random number method, including 83 cases in the control group, 40 males and 43 females, with an age range of 24-70 years and an average age of (48.25 \pm 5.22$)$ years. Traditional nursing management was implemented; There were 84 cases in the observation group, 41 males and 43 females, aged about 22-69 years, with an average age of (46.35 \pm 6.02$)$ years. Information-based nursing management was implemented. The study has passed the review of hospital ethics committee, and there is no significant difference in general data such as basic diseases, age and gender characteristics between the two groups $(\mathrm{P}>0.05)$.

\section{Method}

\subsection{The control group used traditional nursing management.}

3.1.1. The observation group adopted modern information-based nursing management, as follows: Establishment of information nursing management system

Outpatient management system: Combined with the practical experience of hospital daily management, outpatient service is the busiest place and the place where patients spend the longest time. Registration, payment and treatment all need to wait in line for a long time, in order to reduce the workload of outpatient doctors and reduce the treatment time of patients, touch networked computers are installed in the hospital registration office, cashier's office, guide desk and treatment room respectively, so that patients can query the number of doctors on duty and patients at any time, so as to make reasonable choices; After successful registration, the basic information of registered patients will be transmitted from the computer at the registration office to relevant departments, so that the receiving doctors and inspectors can timely understand the basic situation of patients, so as to effectively reduce the waiting time of patients and greatly improve the efficiency of outpatient nursing ${ }^{[2]}$.

Medication management system: After the patient completes the diagnosis and treatment, the patient's medical record information, doctor's orders, prescriptions and other information will be stored in the hospital medication management system. At the same time, the QR code information on the patient's Registration Bill will be connected with the pharmacy management system. When the patient takes the medication, only the QR code on the registration bill needs to be aligned with the window scanner. After reading the QR code information on the Registration Bill.The drug name and dosage on the doctor's order will be stored in the computer, and the drugs will be distributed by themselves, which not only improves the work efficiency, but also ensures the drug safety of patients.

Nurse order processing management system: In order to facilitate nurses to accurately execute doctors' orders, a special doctor's order management system can be set up with the help of Internet information technology. After diagnosing a patient, the doctor will input the doctor's order into the ward computer and transmit it to the computer terminal of the nurse station. The resident nurse station will appear a reminder to process the doctor's order, which is specific to the person. The nurse can verify and confirm the doctor's order through the computer of the nurse station, and then apply for the doctor's order medicine. The processed doctor's orders are printed through the infusion card module to facilitate nurses to execute doctor's orders more accurately, quickly and conveniently, which can significantly improve the efficiency of doctor's order processing ${ }^{[3]}$.

Patient daily care processing system: A unique mode table is set in the patient's daily nursing processing system. When the nurses adjust the information, it is only determined according to the specific situation of the patient, and the patient's information is only collected by the patient's bedside handheld computer, which greatly simplifies the work flow of the nurses and greatly improves the nursing quality. 
Medical record query system:When building the patient medical record information query system, scientifically integrate the computer information technology. Patients can query the specific conditions of various treatment inspection records, charge details and nursing items through hospitalization number and name, so as to clarify the condition and understand the hospitalization expenses.

\subsubsection{Informatization of nursing management}

(1) Improve nursing quality control system

A three-level nursing quality monitoring system of nursing department, department head nurse and department quality control team were established. Department managers and head nurses should check the patient's ward in an all-round way. Once problems are found, they should immediately enter relevant information into the quality control system and check it after rectification to ensure the effective improvement of nursing quality ${ }^{[4]}$.

(2) Electronic nursing medical record management

The head nurse and nursing quality control management personnel of the Department dynamically monitor the qualified rate and quality of patients' electronic nursing medical records, including writing quality, nursing link quality, terminal quality and so on. Proofread the text in the medical record, check whether there are typos, typos, modification, deletion and other problems. At the same time, the head nurse can also check the primary nursing records through random sampling. Before patients are discharged from hospital, nursing quality management is emphasized in order to form a joint, scientific and standardized electronic nursing medical record ${ }^{[5]}$.

(3) Implement the core nursing system

After completing various nursing management work such as doctor's order, the nursing staff shall scan the drug label with PDA and check with the patient's wristband information. After computer scanning, the drug information used by the patient within one day will appear in the doctor's order execution details. During patient drug management, it is only necessary to perform detailed operations according to the doctor's order, it avoids wrong or less drugs.

(4) Nursing records

In order to facilitate the query of nursing records, nurses can record the nursing contents on the nursing records, which can be further improved and supplemented after one day's work. Generally, it cannot be changed after 24 hours. When recording nursing information, nurses are required to treat it carefully and write it correctly, so as to facilitate the succeeding nurses to clearly understand the nursing services received by patients before.

(5) Implementation of evaluation system

After receiving the patients in the ward, the responsible nurse needs to conduct a comprehensive and detailed evaluation of the specific situation of the patients. If the receiving patients are high-risk patients, they need to be evaluated once or twice a week. Once the patient's condition changes during the treatment, they need to be evaluated at any time. The head nurse needs to evaluate the accuracy of the patient's condition evaluation completed by the nursing staff every week ${ }^{[6]}$.

\subsection{Observation indicators}

The effects of patient nursing management, basic nursing, ward management, specialized nursing and nursing satisfaction under the two nursing methods were compared.

\subsection{Statistical methods}

SPSS19.0 statistical software was used to analyze and process the relevant data. The counting data was 
expressed in \%, the comparison was expressed by $\mathrm{X}^{2}$ test, and the measurement data was $\bar{x} \pm s$ expressed by $\mathrm{t}$ test. The difference was statistically significant $(\mathrm{P}<0.05)$.

\section{Results}

\subsection{Comparison of clinical nursing effects between the two groups}

The effective rate of clinical nursing under the two nursing modes was $98.80 \%$ in the observation group and $90.36 \%$ in the control group. The $\mathrm{x} 2$ test result was 4.3043 . The effectiveness of the observation group was significantly higher than that of the control group, and $\mathrm{P}<0.001$. See Table 1.

Table 1. The clinical nursing effects of the two groups were compared [n (\%)]

\begin{tabular}{llllll}
\hline Group & Recovery & $\begin{array}{l}\text { Remarkable } \\
\text { effect }\end{array}$ & Better & Invalid & Efficient \\
\hline $\begin{array}{l}\text { Control group }(\mathrm{n}=28(33.73) \\
83)\end{array}$ & $39(46.98)$ & $8(9.63)$ & $8(9.63)$ & $75(90.36)$ \\
$\begin{array}{l}\text { Observation group } \\
(\mathrm{n}=84)\end{array}$ & & & & & \\
$\mathrm{X}^{2}$ & & & & & \\
$\mathrm{P}$ & & & & & \\
\hline
\end{tabular}

\subsection{Comparison of nursing satisfaction between the two groups}

Compared with the control group, the nursing satisfaction of patients in the observation group was significantly higher than that in the control group $(98.80 \%>86.74 \%)$, the difference was statistically significant $(\mathrm{P}<0.05)$. See Table 2.

Table 2. Survey and comparison of patients' nursing satisfaction [n (\%)]

\begin{tabular}{|c|c|c|c|c|c|}
\hline Group & Number of cases & Very satisfied & Satisfied & Dissatisfied & Total satisfaction \\
\hline control group & 83 & $30(36.14)$ & $42(50.60)$ & $11(13.25)$ & $72 / 86.74$ \\
\hline $\begin{array}{l}\text { Observation } \\
\text { group }\end{array}$ & 84 & $54(64.28)$ & $29(34.52)$ & $1(1.19)$ & $83 / 98.80$ \\
\hline $\mathrm{X}^{2}$ & & & & & 9.1083 \\
\hline $\mathrm{P}$ & & & & & 0.0025 \\
\hline
\end{tabular}

\section{Discussion}

With the further advancement of China's medical and health reform, it is urgent to apply modern information technology science to hospital nursing management, and establish a perfect outpatient management system, electronic medical record management system, doctor's order execution system and patient's daily nursing processing system in combination with cloud computing technology and computer network technology, It not only facilitates the normal development of nursing staff's daily nursing management, but also plays a positive role in improving nursing efficiency, improving patient nursing effect and improving patient nursing satisfaction. Combined with clinical trial practice, this study conducted a targeted nursing model study on 167 selected patients. It was found that under the application of information nursing model, $86.74 \%$ of the patients; In terms of nursing effect, among the 84 patients in the observation group, only 1 was ineffective, and the nursing effective rate was $98.80 \%$, while among the 83 patients in the control group, 8 were ineffective, and the nursing effective rate was only $90.36 \%$, which fully verified 
the clinical application value of information-based nursing management. In addition, the scientific application of information-based nursing management can significantly improve the work efficiency of nursing staff.

The main reasons are as follows:

(1) Through the construction of relevant information systems, the procedures of patient registration, waiting and payment can be simplified, the investment of hospital human resources can be reduced, and the waiting time can be shortened.

(2) Information management has effectively reduced the workload of nursing staff, and the operation of relevant information systems has replaced the manual work in the past, such as registration, waiting and so on. At the same time, the information system can accurately reflect the situation of doctors on duty, so that patients have more diversified choices.

(3) In the past, some work with poor professionalism and low technical content required nurses to complete, but after the introduction of information system, patients and their families can complete the operation through the system.

By optimizing the nursing process and saving working time, nurses have enough time to study nursing technology and knowledge, so they are handier in completing various nursing tasks, and the nursing quality and nursing satisfaction have been effectively improved.

\section{Disclosure statement}

The author declares no conflict of interest.

\section{References}

[1] Chen G, Xiao S, Qiu L, Ke J, Gu M. Effect analysis of nursing management in grass-roots hospitals based on informatization [J]. Modern hospital, 2021, 19 (07):31-33.

[2] Bi H. On the application of informatization in TCM nursing management [J]. Yunnan Journal of traditional Chinese medicine, 2016, 32 (11): 90.

[3] Xie P, Sun X. Application effect of mobile nurse information management in optimizing nursing human resource management [J]. Nursing research, 2020, 34 (3): 514-516.

[4] Zhang L, Xue X, Wei S, et al. Information analysis and function design of nursing management system [J]. China Digital Medicine, 2018, 13 (7): 55-57.

[5] Wu C, Xi M, Tan C, et al. Construction and application of intelligent management system for nursing quality [J]. China health quality management, 2017, 24 (5): 81-83.

[6] Cheng H, Liu J. Cause analysis and Countermeasures of hindering the reporting of nursing adverse events [J]. Nursing research, 2018, 32 (14): 2294-2296. 